\title{
Knowns and Unknowns: the Safety and Efficacy of Cancer Immunotherapy in Chronic Liver Disease
}

\author{
David M. Chascsa ${ }^{1} \cdot$ Jorge Rakela $^{1}$ \\ Published online: 21 June 2018 \\ (C) Springer Science+Business Media, LLC, part of Springer Nature 2018
}

Immunotherapies are quickly revolutionizing the field of oncology and have shown great success and promise in many subtypes of malignancy including liver cancer. Studies suggest that significant survival benefit can be achieved in treating melanoma, lung cancer, and possibly hepatocellular carcinoma (HCC). However, data are extremely limited with respect to the implications of use in patients with chronic liver disease. Immunotherapeutic agents which include targets of programmed cell death protein (PD), PD-L1, and cytotoxic T lymphocyte-associated protein 4 (CTLA-4) inhibitors work by blocking inhibition of the immune system, thwarting cancers' evasive mechanisms to hide from immune recognition and thus escape immune-mediated destruction [1]. However, these therapies can also ramp up the immune system and may predispose to organ immunogenicity, including immuneinduced hepatitis. Reports suggest that hepatotoxicity is rare, occurring on the order of 4-15\%, which may make liver injury less common with these agents compared with traditional chemotherapeutic agents, and is less than the reported gastrointestinal toxicities such as immunotherapy-induced colitis [2, 3]. CTLA-4 inhibitors have been associated with increased risk compared with PD-1 inhibitors [4]. However, PD-1/PDL1 inhibitors are beginning to be used in hepatocellular carcinoma treatment and the reported grades 3-4 toxicities including serum aminotransferase elevation, bilirubin rise, and pruritus may occur in nearly $25 \%$ of patients undergoing treatment [5]. Combination immunotherapy appears to also increase the risk of hepatotoxicity beyond that of single agents [6]. There is a vast variability in hepatotoxicity ranging from asymptomatic mild transaminase increases to fulminant hepatic failure and death, which has been described in post-liver

David M. Chascsa

Chascsa.david@mayo.edu

1 Division of Gastroenterology and Hepatology, Mayo Clinic, 5777 E. Mayo Blvd, Phoenix, AZ 85054, USA transplant patients [7-11]. Interestingly, these agents have also saved patients with high metastatic hepatic tumor burden from death by staving off acute liver failure [12].

Data are still very limited regarding the tolerability of immune-checkpoint inhibitors for patients with underlying liver disease. There are reports of those patients with hepatitis $\mathrm{B}$ and $\mathrm{C}$ being treated and tolerating therapy without developing immune-mediated hepatitis [10]. However, there is likely bias with respect to the use of these agents in patients with underlying liver disease as initial studies excluded patients with significant liver disease or history of organ transplantation. With the promise of improved outcomes in the treatment of HCC, including disease control rates in excess of $50 \%$, more patients with chronic underlying liver disease will potentially be exposed to immunotherapy [11]. Checkpoint inhibitor targets are expressed on HCC and their levels are not altered by underlying liver disease [13]. In fact, expression may be upregulated [14]. Nivolumab, a PD-1 inhibitor, is approved for use in patients with HCC with inadequate response to sorafenib. Pembrolizumab, also a PD-1 inhibitor, has shown efficacy in HCC including in immunosuppressed post-liver transplant recipients [15]. However, other transplant patients treated for HCC and other metastatic malignancies have developed allograft rejection which in some cases was fatal. Therapeutic trials are ongoing to determine the efficacy of immune-checkpoint inhibitor therapy in cholangiocarcinoma, which may expose patients with an underlying immunemediated liver disease such as primary sclerosing cholangitis to immunotherapy [16].

The pattern of hepatic injury from checkpoint inhibitors is unique compared with that of other cancer chemotherapeutic agents. While traditional agents are associated with a pattern of drug-induced liver injury, immunotherapies present with features of immune-mediated hepatitis. The presentation of hepatic injury can be quite variable from very low-level transient rises in liver tests to severe elevations in alanine aminotransferase (ALT) and aspartate aminotransferase (AST) levels. The pattern of injury is largely hepatocellular as 
opposed to cholestatic. Histologically, there is a pan-lobular hepatitis with neutrophilic and lymphocytic infiltrates which can mimic the findings of autoimmune hepatitis [17]. The differentiating factor from de novo autoimmune hepatitis is the lack of plasma cell predominance in the lymphocytic infiltrate. While bile duct injury has been reported, it is not a hallmark feature of immunotherapy-induced injury.

Treatment of immunotherapy-induced hepatitis by and large consists of wrangling in and quieting down the immune system activation induced by these therapies. Liver injury may not become apparent after the first dose of therapy and may occur only after additional cycles. While many instances of mild liver test elevations will resolve spontaneously, ongoing liver injury necessitates treatment [18]. A step-up approach is usually recommended in a manner similar to the treatment of autoimmune hepatitis [19]. First-line therapy utilizes pulsed corticosteroids. Failure to respond to monotherapy should prompt consideration of additional, typically oral, treatment with mycophenolic acid preparations [20]. As the pharmacologic mechanism for these drugs is activation of $\mathrm{T}$ cellmediated pathways, refractory immunotherapy-induced hepatitis may necessitate the use of anti-thymocyte globulin, a T cell-depleting agent [21]. This therapy carries the risk of negating the therapeutic effect of these drugs. Notably, there is one strategic difference with regard to the treatment of hepatitis compared with the GI toxicities of immunotherapy drugs. While anti-tumor necrosis factor (anti-TNF) drugs such as infliximab are used to treat immune-mediated colitis, they are typically not used in checkpoint inhibitor-induced hepatitis given their own propensity to cause hepatic injury. After successful treatment, maintenance prednisone may be required, which may allow for continuation of the immunotherapy drug. However, severe cases of hepatitis will likely not justify the risk of repeated drug challenge.

Liver transplant patients are a unique population who rely on adequate control of the immune system to prevent allograft rejection. While there are significant reports of allograft rejection in the kidney transplant population, there are fewer cases of liver allograft rejection reported in the literature. In fact, there are several case reports suggesting tolerability [22-24]. Perhaps this finding is due to the more immune-tolerant nature of the liver as compared with other organs, and the liver's ability to essentially induce immune hypo-responsiveness [25]. However, there is high concern for publication bias, and there are certainly growing numbers of reports of liver allograft rejection in the setting of immunotherapy. Theoretically, the amount of immunosuppression required to prevent chronic allograft rejection could inhibit the response of checkpoint inhibitor chemotherapy. Case reports are mixed regarding the benefit of immunotherapy in the setting of chronic immunosuppression with some suggesting no significant response and others suggesting quite good responses. Immunosuppressive regimens may be altered in the setting of malignancy. Given that immune system inhibition is associated with more rapid tumor growth, patients may be on less or even minimal immunosuppression at the time immunotherapy is considered. It has been suggested that calcineurin inhibitors may reduce efficacy and consideration should be given to transitioning patients to mammalian target of rapamycin (mTOR) inhibitors [23]. Great caution should be taken when considering and initiating immunotherapy in liver transplant patients as irreversible allograft rejection has been reported. As in non-transplant patients, treatment of this complication may require steroids, mycophenolic acid, or anti-thymocyte globulin [26]. While successful reversal of allograft rejection is possible and reported, immunotherapy-induced allograft rejection should negate attempts at repeated drug challenge as re-transplantation will likely not be an available treatment option as these agents are used for the treatment of active uncontrolled malignancy which is typically a contraindication to transplant.

In summary, cancer immunotherapy has shown great promise for the treatment of a multitude of malignancies with impressive results. While it is known these agents can induce immune-mediated hepatic injury, their overall safety has not been fully elucidated in the setting of chronic liver disease. Significant hepatic injury and death have been reported, especially in the setting of liver transplant. The results from larger studies assessing efficacy and safety of these medications in the setting of chronic liver disease are eagerly awaited.

Author Contributions DMC composed the manuscript. JR provided critical review of the manuscript.

\section{Compliance with Ethical Standards}

Conflict of Interest David M. Chascsa and Jorge Rakela declare no conflicts of interest.

Human and Animal Rights and Informed Consent This article does not contain any studies with human or animal subjects performed by any of the authors.

\section{References}

1. Salmaninejad A, Khoramshahi V, Azani A, Soltaninejad E, Aslani S, Zamani MR, et al. PD-1 and cancer: molecular mechanisms and polymorphisms. Immunogenetics. 2018;70(2):73-86.

2. Cramer P, Bresalier RS. Gastrointestinal and hepatic complications of immune checkpoint inhibitors. Curr Gastroenterol Rep. 2017;19(1):3.

3. Kim KW, Ramaiya NH, Krajewski KM, Jagannathan JP, Tirumani SH, Srivastava A, et al. Ipilimumab associated hepatitis: imaging and clinicopathologic findings. Investig New Drugs. 2013;31(4): 1071-7.

4. Wang W, Lie P, Guo M, He J. Risk of hepatotoxicity in cancer patients treated with immune checkpoint inhibitors: a systematic review and meta-analysis of published data. Int J Cancer. 2017;141(5):1018-28. 
5. Long J, Lin J, Wang A, Wu L, Zheng Y, Yang X, et al. PD-1/PD-L blockade in gastrointestinal cancers: lessons learned and the road toward precision immunotherapy. Journal of Hematology and Oncology. 2017;10(1):146.

6. Puzanov I, Diab A, Abdallah K, et al. Managing toxicities associated with immune checkpoint inhibitors: consensus recommendations from the Society for Immunotherapy of Cancer (SITC) Toxicity Management Working Group. J Immunother Cancer. 2017;5(1):95.

7. Tanaka R, Fujisawa Y, Sae I, Maruyama H, Ito S, Hasegawa N, et al. Severe hepatitis arising from ipilimumab administration, following melanoma treatment with nivolumab. Jpn J Clin Oncol. 2017;47(2):175-8.

8. Tang C, Welsh JW, de Groot P, Massarelli E, Chang JY, Hess KR, et al. Ipilimumab with stereotactic ablative radiation therapy: phase I results and immunologic correlates from peripheral T cells. Clin Cancer Res. 2017;23(6):1388-96.

9. Spankuch I, Gassenmaier M, Tampouri I, et al. Severe hepatitis under combined immunotherapy: resolution under corticosteroids plus antithymocyte immunoglobulins. Eur J Cancer. 2017;81:203-5.

10. Rai R, Ezeoke OM, McQuade JL, et al. Immunotherapy in patients with concurrent solid organ transplant, HIV, and hepatitis B and C. Ann Oncol. 2017;28(Supplement 5):v408.

11. El-Khoueiry AB, Sangro B, Yau T, et al. Nivolumab in patients with advanced hepatocellular carcinoma (CheckMate 040): an open-label, non-comparative, phase 1/2 dose escalation and expansion trial. Lancet. 2017;389(10088):2492-502.

12. Sandoval-Sus JD, Mogollon-Duffo F, Patel A, Visweshwar N, Laber DA, Kim R, et al. Nivolumab as salvage treatment in a patient with HIV-related relapsed/refractory Hodgkin lymphoma and liver failure with encephalopathy. J Immunother Cancer. 2017;5:49.

13. Xing D, Luan L, Zhu Q, et al. PD-L1 and LAG3 expression in hepatocellular carcinoma associated with $\mathrm{HCV}$ and steatohepatitis. Lab Investig. 2017;97(Supplement 1):426A.

14. Zhou G, Sprengers D, Boor PPC, Doukas M, Schutz H, Mancham $\mathrm{S}$, et al. Antibodies against immune checkpoint molecules restore functions of tumor-infiltrating T cells in hepatocellular carcinomas. Gastroenterology. 2017;153(4):1107-1119.e1110.

15. Rammohan A, Reddy MS, Farouk M, Vargese J, Rela M. Pembrolizumab for metastatic hepatocellular carcinoma following live donor liver transplantation: the silver bullet? Hepatology. 2018;67(3):1166-8.

16. Rizvi S, Khan SA, Hallemeier CL, Kelley RK, Gores GJ. Cholangiocarcinoma-evolving concepts and therapeutic strategies. Nat Rev Clin Oncol. 2018;15(2):95-111.

17. Kleiner DE, Berman D. Pathologic changes in ipilimumab-related hepatitis in patients with metastatic melanoma. Dig Dis Sci. 2012;57(8):2233-40.

18. Gangadhar TC, Vonderheide RH. Mitigating the toxic effects of anticancer immunotherapy. Nat Rev Clin Oncol. 2014;11(2):91-9.

19. De Martin E, Michot JM, Papouin B, et al. Characterization of liver injury induced by cancer immunotherapy using immune checkpoint inhibitors. J Hepatol. 2018;68(6):1181-90.

20. Spain L, Diem S, Larkin J. Management of toxicities of immune checkpoint inhibitors. Cancer Treat Rev. 2016;44:51-60.

21. Cheng R, Cooper A, Kench J, Watson G, Bye W, McNeil C, et al. Ipilimumab-induced toxicities and the gastroenterologist. $\mathrm{J}$ Gastroenterol Hepatol. 2015;30(4):657-66.

22. Biondani P, De Martin E, Samuel D. Safety of an anti-PD-1 immune checkpoint inhibitor in a liver transplant recipient. Ann Oncol. 2018;29(1):286-7.

23. Maggiore U, Pascual J. The bad and the good news on cancer immunotherapy: implications for organ transplant recipients. Adv Chronic Kidney Dis. 2016;23(5):312-6.

24. Varkaris A, Lewis DW, Nugent FW. Preserved liver transplant after PD-1 pathway inhibitor for hepatocellular carcinoma. Am J Gastroenterol. 2017;112(12):1895-6.

25. Taner T, Hansen M, Park W, Stegall M. Phenotypic and functional assessment of $\mathrm{t}$ cell alloimmunity after liver transplantation. Am J Transplant. 2017;17(Supplement 3):528.

26. Ahmed T, Pandey R, Shah B, Black J. Resolution of ipilimumab induced severe hepatotoxicity with triple immunosuppressants therapy. BMJ Case Rep. Jul 14;2015. https://doi.org/10.1136/bcr-2014208102. 